\title{
Some connections between binary block codes and Hilbert algebras
}

\author{
Cristina FLAUT \\ Faculty of Mathematics and Computer Science, \\ Ovidius University, \\ Bd. Mamaia 124, 900527, Constanţa, \\ România \\ cflaut@univ-ovidius.ro \\ cristina_flaut@yahoo.com \\ http://cristinaflaut.wikispaces.com/ \\ http://www.univ-ovidius.ro/math/
}

\begin{abstract}
In this paper, we will study some connections between Hilbert algebras and binary block-codes. With these codes, we can eassy obtain orders which determine suplimentary properties on these algebras. We will try to emphasize how, using binary block-codes, we can provide examples of classes of Hilbert algebras with some properties, in our case, classes of semisimple Hilbert algebras and classes of local Hilbert algebras.
\end{abstract}

Keywords. BCK-algebras; Hilbert algebras; Block codes.

AMS Classification. 06F35.

\section{Introduction}

Over the last years, codes have experienced a significant development. Using codes, an impressive quantity of data can be transmitted. They have important applications in various domains with implications in social life. Using codes, data are trasformed into a form which can be easily understand by computer software and can be represented in a form which is more resistant to errors in data transmission or data storage. In this way, data, in their quantitative or qualitative forms, are classified to facilitate some analysis.

Coding Theory is a mathematical domain with many applications in Information Theory. Various type of codes and their connections with other mathematical objects have been intensively studied. One of these applications, namely 
connections between binary block codes and BCK-algebras, was recently studied in (Jun, Song, 2011), (Flaut, 2015) and (Borumand Saeid, Fatemidokht, Flaut, Kuchaki Rafsanjani, 2015). Starting from these results and since a positive implicative BCK-algebra is a Hilbert algebra, in this paper we will study some connections between Hilbert algebras and binary block-codes. Even if in (Borumand Saeid, Fatemidokht, Flaut, Kuchaki Rafsanjani, 2015) and (Chajda, Halaš, Jun, 2002) it was remarked that a BCK-algebra and a Hilbert algebra can be obtained on any ordered set with a greatest element $\theta$, using codes we can easily obtain orders which determine suplimentary properties of these algebras. In the following, we will try to emphasize how, using binary block-codes, we can provide examples of classes of Hilbert algebras with certain properties, in our case, classes of semisimple Hilbert algebras (Theorem 3.11) and classes of local Hilbert algebras (Theorem 3.12). Hilbert algebras were first introduced at the middle of the 20th century and they are an important tool for some investigations in intuitionistic logics and other non-classical logics (see (Piciu, Buşneag, 2010)).

\section{Preliminaries}

Definition 2.1. An algebra $(X, *, \theta)$ of type $(2,0)$ is called a $B C I$-algebra if the following conditions are fulfilled:

1) $((x * y) *(x * z)) *(z * y)=\theta$, for all $x, y, z \in X$;

2) $(x *(x * y)) * y=\theta$, for all $x, y \in X$;

3) $x * x=\theta$, for all $x \in X$;

4) For all $x, y, z \in X$ such that $x * y=\theta, y * x=\theta$, it results $x=y$.

If a BCI-algebra $X$ satisfies the following identity:

5) $\theta * x=\theta$, for all $x \in X$, then $X$ is called a $B C K$-algebra.

A BCK-algebra $X$ is called commutative if $x *(x * y)=y *(y * x)$, for all $x, y \in X$ and implicative if $x *(y * x)=x$, for all $x, y \in X$. A BCK-algebra $(A, *, 0)$ is called positive implicative if and only if

$$
(x * y) * z=(x * z) *(y * z), \text { for all } x, y, z \in A .
$$

The partial order relation on a BCK-algebra is defined such that $x \leq y$ if and only if $x * y=\theta$.

Remark 2.2. The following BCK-algebra $(X, *, \theta)$

$$
\begin{gathered}
\theta * x=\theta \text { and } x * x=\theta, \forall x \in X \\
x * y=\theta, \text { if } x \leq y, \quad x, y \in X \\
x * y=x, \text { otherwise }
\end{gathered}
$$

is a non-commutative and a non-implicative algebra. (see (Diego,1966) and (Flaut, 2015))

Definition 2.3. A Hilbert algebra is a triplet $(H, *, 1)$ in which $H$ is a non-empty set, "*" a binary operation on $H$ and $1 \in H$ is a fixed element such that the following relations hold, for all $x, y, z \in H$ : 
1) $x *(y * x)=1$;

2) $(x *(y * z)) *((x * y) *(x * z))=1$;

3) $x * y=1$ and $y * x=1$ imply $x=y$.

Proposition 2.4. ((Dudek, 1999), Theorem) An algebra $(H, *, \theta)$ is a Hilbert algebra if and only if its dual algebra $(H, \cdot \theta)$, where $x \cdot y=y * x$, is a positive implicative BCK-algebra.

Definition 2.5. Let $(H, \cdot, \theta)$ be a Hilbert algebra. A subset $L$ of the algebra $H$ is called a filter (or implicative filter) of $H$ if we have $\theta \in L$ and if for all $x, y \in L$, from $x \in L$ and $x \cdot y \in L$ it results that $y \in L$.

Definition 2.6. (see (Buşneag, 1987)) Let $H$ be a Hilbert algebra.

1) Let $L$ be a filter of the algebra $H$. $L$ is called maximal if for a proper filter $F$ of $H$ if $L \subseteq F$, we have $L=F$.

2) $H$ is called a semisimple Hilbert algebra if the intersection of all maximal filters of $H$ is $\{\theta\}$.

3) $H$ is called a local algebra if and only if the algebra $H$ contains only one maximal filter.

For other details about Hilbert algebras, the reader is referred to ( Buşneag, 1987), (Dan, 2008).

\section{Main results}

Proposition 3.1. BCK-algebra $(X, *, \theta)$ defined by the relation (2.1.) is a positive implicative algebra.

Proof. We must prove that $(x * y) * z=(x * z) *(y * z)$, for all $x, y, z \in X$.

Case 1: at least one element is $\theta$.

i) $(\theta * x) * z=\theta * z=\theta$ and $(\theta * z) *(x * z)=\theta *(x * z)=\theta$;

ii) $(x * \theta) * z=x * z=x$ and $(x * z) *(\theta * z)=x * \theta=x$;

iii) $(x * y) * \theta=x * y=x$ and $(x * \theta) *(y * \theta)=x * y=x$;

Case 2: one element is comparable with another.

i) $x \leq y$; $(x * y) * z=\theta * z=\theta$ and $(x * z) *(y * z)=x * y=\theta$;

ii) $x \leq z ;(x * y) * z=x * z=\theta$ and $(x * z) *(y * z)=\theta * y=\theta$;

iii) $z \leq y$; $(x * y) * z=x * z=x$ and $(x * z) *(y * z)=x * y=x$;

iv) $y \leq x ;(x * y) * z=x * z=x$ and $(x * z) *(y * z)=x * y=x$;

v) $z \leq x ;(x * y) * z=x * z=x$ and $(x * z) *(y * z)=x * y=x$;

vi) $y \leq z ;(x * y) * z=x * z=x$ and $(x * z) *(y * z)=x * \theta=x$;

Case 3: two elements are comparable with the third.

$x \leq y$ and $z \leq y ;(x * y) * z=\theta * z=\theta$ and $(x * z) *(y * z)=x * y=\theta$,etc.

Remark 3.2. Let $v_{x}=x_{1} x_{2} \ldots x_{n}$ and $v_{y}=y_{1} y_{2} \ldots y_{n}$ be two codewords belonging to a binary block-code $V$. We define an order relation $\leqslant_{c}$ on the set of codewords belonging to a binary block-code $V$, as follows ( see (Jun, Song, 2011)): 


$$
v_{x} \leqslant c v_{y} \Leftrightarrow y_{i} \leqslant x_{i} \text { for } i=1,2, \ldots, n \text {. }
$$

Definition 3.3. (Jun, Song, 2011)

i) A mapping $f: A \rightarrow X$ is called a $B C K$-function on $A$. A cut function of $f$ is a map $f_{r}: A \rightarrow\{0,1\}, r \in X$, such that $f_{r}(x)=1$, if and only if $r * f(x)=\theta, \forall x \in A$.

A cut subset of $A$ is the following subset of $A, A_{r}=\{x \in A: r * f(x)=\theta\}$.

ii) Let $A=\{1,2, \ldots, n\}$ and let $X$ be a BCK-algebra. For each BCKfunction $\tilde{A}: A \rightarrow X$ a binary block-code of length $n$ was defined. A codeword in a binary block-code $V$ is $v_{x}=x_{1} x_{2} \ldots x_{n}$ such that $x_{i}=x_{j} \Leftrightarrow A_{x}(i)=j$, for $i \in A$ and $j \in\{0,1\}$.

Let $X$ be a BCK-algebra. Let $V$ be a binary block-code with $n$ codewords of length $n$. We consider the matrix $M_{V}=\left(m_{i, j}\right)_{i, j \in\{1,2, \ldots, n\}} \in \mathcal{M}_{n}(\{0,1\})$ with the rows consisting of the codewords of $V$. This matrix is called the matrix associated to the code $V$.

Let $C$ be a binary block code with $n$ codewords of length $m$. From Proposition 3.8 and Theorem 3.9 from (Flaut, 2015), we find a BCK-algebra $X$ such that the obtained binary block-code $V_{X}$ contains the binary block-code $C$ as a subset. In the following, we briefly present this procedure.

Let $V$ be a binary block-code, $V=\left\{x_{1}, x_{2}, \ldots, x_{n}\right\}$, with codewords of length $m$. We consider the codewords $x_{1}, x_{2}, \ldots, x_{n}$ lexicographically ordered, $x_{1} \geq_{\text {lex }}$ $x_{2} \geq_{\text {lex }} \ldots \geq_{\text {lex }} x_{n}$. Let $M \in \mathcal{M}_{n, m}(\{0,1\})$ be the associated matrix with the rows $w_{1}, \ldots, w_{n}$ in this order. We can extend the matrix $M$ to a square matrix $M^{\prime} \in \mathcal{M}_{p}(\{0,1\}), p=n+m$, such that $M^{\prime}=\left(m_{i, j}^{\prime}\right)_{i, j \in\{1,2, \ldots, p\}}$ is an upper triangular matrix with $m_{i i}=1$, for all $i \in\{1,2, \ldots, p\}$. For this purpose, we insert in the left side of the matrix $M$ (from the right to the left) the following $n$ new columns of the form $\underbrace{00 \ldots 01}_{n}, \underbrace{00 \ldots 10}_{n}, \ldots, \underbrace{10 \ldots 00}_{n}$. A new matrix $D$ with $n$ rows and $n+m$ columns results. Now, we insert at the bottom of the matrix $D$ the following $m$ rows: $\underbrace{00 \ldots 010 \ldots 00}_{n}, \underbrace{00 \ldots 001 \ldots 00}_{m+1}, \ldots, \underbrace{000}_{m-1} 1$. We obtain the required matrix $M^{\prime}$. If the first line of the matrix $M^{\prime}$ is not $\underbrace{11 \ldots 1}_{p}$, then we insert the row $\underbrace{11 \ldots 1}_{p+1}$ as a first row and the column $\underbrace{10 \ldots 0}_{p}$ as a first column. We obtain a new code $W=\left\{\theta, w_{1}, \ldots, w_{n+m}\right\}$. Using relation $(2.1)$, we define on $\left(W, \leqslant_{c}\right)$ a binary relation " *". It results that $X=(W, *, \theta)$ becomes a BCK-algebra.

Proposition 3.4. Let $C$ be a binary block code with $n$ codewords of length $m$. With the above notations, we have that $\left\{\theta, w_{n+1}, \ldots, w_{n+m}\right\}$ determines a filter in the Hilbert algebra $(X, \cdot, \theta)$, obtained in Remark 3.2.

Proof. It is obvious.

Definition 3.5. A mapping $\tilde{A}: A \rightarrow X$ is called an $H$-function on $A$, where $A$ and $X$ are a nonempty set and $X$ is a Hilbert-algebra, respectively. 
Definition 3.6. A cut function of $\tilde{A}$, for $q \in X$, where $X$ is a Hilbert algebra, is defined to be a mapping $\tilde{A}_{q}: A \rightarrow\{0,1\}$ such that $(\forall x \in A)\left(\tilde{A}_{q}(x)=\right.$ $1 \Leftrightarrow q * \tilde{A}(x)=0)$.

Definition 3.7. Let $A=\{1,2, \ldots, n\}$ and let $X$ be a Hilbert-algebra. A codeword in a binary block-code $V$ is $v_{x}=x_{1} x_{2} \ldots x_{n}$ such that $x_{i}=x_{j} \Leftrightarrow$ $\widetilde{A}_{x}(i)=j$ for $i \in A$ and $j \in\{0,1\}$.

Proposition 3.8. Let $C$ be a binary block code with $n$ codewords of length $m$ and let $X$ be the associated Hilbert algebra, as in the above. Therefore, there are the sets $A$ and $B \subseteq X$, the H-function $f: A \rightarrow X$ and a cut function $f_{r}$ such that

$C=\left\{f_{r}: A \rightarrow\{0,1\} / f_{r}(x)=1\right.$, if and only if $\left.r * f(x)=\theta, \forall x \in A, r \in B\right\}$

Proposition 3.9. (Flaut, 2015, Remark 3.6.) If $\mathfrak{N}_{n}$ is the number of all finite non-isomorphic BCK-algebras with $n$ elements, then $\mathfrak{N}_{n} \geq 2^{\frac{(n-1)(n-2)}{2}}$.

Proposition 3.10. If $\mathcal{N}$ is the number of all finite non-isomorphic Hilbert algebras $(X, \cdot, \theta)$ with $n$ elements, then $\mathcal{N} \geq 2^{\frac{(n-1)(n-2)}{2}}$.

Proof. From Remark 2.1, we know that the multiplication "* "given in relation (2.1) defines on the $(X, *, \theta)$ a structure of a positive implicative BCKalgebra. Since to each positive implicative BCK-algebra corresponds a Hilbert algebra, we will use Proposition 2.4 and we obtain the required result.

Theorem 3.11. Let $V$ be a binary block-code with $n$ codewords of length $n, V=\left\{x_{1}, x_{2}, \ldots, x_{n}\right\}$. We consider the codewords $x_{1}, x_{2}, \ldots, x_{n}$ lexicographically ordered, $x_{1} \geq_{\text {lex }} x_{2} \geq_{\text {lex }} \ldots \geq_{\text {lex }} x_{n}$ such that $x_{1}=\underbrace{11 \ldots 1}_{n}, x_{2}=\underbrace{010 \ldots 0}_{n}, x_{3}=$ $\underbrace{001 \ldots 0}_{n}, x_{n}=\underbrace{000 \ldots 1}_{n}$. With the above notations, the Hilbert algebra $(X, \cdot, \theta)$, obtained as in Remark 3.2, is a semisimple Hilbert algebra.

Proof. The algebra $X$ has the following elements $X=\left\{\left(\theta=x_{1}\right), x_{2}, \ldots, x_{n}\right\}$. Using the construction of this algebra, we obtain that $x_{i} \cdot x_{j}=x_{j}$, for all $i, j \in$ $\{1,2, \ldots, n\}, i \neq j$, therefore, for each $i \neq 1$, we have that $L_{i}=\left\{x_{j} \in X \quad \mid j \neq i\right\}$ is a maximal proper filter in $X$. Since $\underset{i \neq 1}{\cap} L_{i}=\{\theta\}$, it results that $X$ is a semisimple Hilbert algebra.

Theorem 3.12. Let $V$ be a binary block-code with $n$ codewords of length $n, V=\left\{x_{1}, x_{2}, \ldots, x_{n}\right\}$. We consider the codewords $x_{1}, x_{2}, \ldots, x_{n}$ lexicographically ordered, $x_{1} \geq_{\text {lex }} x_{2} \geq_{\text {lex }} \ldots \geq_{\text {lex }} x_{n}$. If the associated matrix $M_{V}=$ $\left(m_{i, j}\right)_{i, j \in\{1,2, \ldots, n\}} \in \mathcal{M}_{n}(\{0,1\})$ of the code $V$ is upper triangular, with $m_{i i}=1$, for all $i \in\{1,2, \ldots, n\}$, and the last column is equal to $\underbrace{11 \ldots 1}_{n}$, with the above notations, the Hilbert algebra $(X, \cdot, \theta)$, obtained as in Remark 3.2, is a local Hilbert algebra.

Proof. The algebra $X$ has the following elements $X=\left\{\left(\theta=x_{1}\right), x_{2}, \ldots, x_{n}\right\}$. Using the construction of this algebra, since $x_{i} \cdot x_{j}=x_{j}$ or $\theta$, and $x_{n} \cdot x_{j}=\theta$, 
for all $j \in\{1,2, \ldots, n\}$, we obtain that $L=\left\{\theta, x_{2}, \ldots, x_{n-1}\right\}$ is the only maximal proper filter in $X$, therefore $X$ is a local Hilbert algebra.

\section{Examples}

Example 4.1. i) Let $C=\{0000,0001,0010,0011\}=\left\{w_{6}, w_{7}, w_{8}, w_{9}\right\}$ be a linear binary block code and let $X=\left\{\theta, w_{2}, w_{3}, w_{4}, w_{5}, w_{6}, w_{7}, w_{8}, w_{9}\right\}$ be the obtained BCK-algebra. The multiplication "*" of this algebra is given in the table below ( see (Borumand Saeid, Fatemidokht, Flaut, Kuchaki Rafsanjani, 2015)):

\begin{tabular}{|l|l|l|l|l|l|l|l|l|l|}
\hline$*$ & $\theta$ & $w_{2}$ & $w_{3}$ & $w_{4}$ & $w_{5}$ & $w_{6}$ & $w_{7}$ & $w_{8}$ & $w_{9}$ \\
\hline$\theta$ & $\theta$ & $\theta$ & $\theta$ & $\theta$ & $\theta$ & $\theta$ & $\theta$ & $\theta$ & $\theta$ \\
\hline$w_{2}$ & $w_{2}$ & $\theta$ & $w_{2}$ & $w_{2}$ & $w_{2}$ & $\mathbf{w}_{2}$ & $\mathbf{w}_{2}$ & $\theta$ & $\theta$ \\
\hline$w_{3}$ & $w_{3}$ & $w_{3}$ & $\theta$ & $w_{3}$ & $w_{3}$ & $\mathbf{w}_{3}$ & $\mathbf{w}_{3}$ & $\theta$ & $\mathbf{w}_{3}$ \\
\hline$w_{4}$ & $w_{4}$ & $w_{4}$ & $w_{4}$ & $\theta$ & $w_{4}$ & $\mathbf{w}_{4}$ & $\mathbf{w}_{4}$ & $\mathbf{w}_{4}$ & $\theta$ \\
\hline$w_{5}$ & $w_{5}$ & $w_{5}$ & $w_{5}$ & $w_{5}$ & $\theta$ & $\mathbf{w}_{5}$ & $\mathbf{w}_{5}$ & $\mathbf{w}_{5}$ & $\mathbf{w}_{5}$ \\
\hline$w_{6}$ & $w_{6}$ & $w_{6}$ & $w_{6}$ & $w_{6}$ & $w_{6}$ & $\theta$ & $w_{6}$ & $w_{6}$ & $w_{6}$ \\
\hline$w_{7}$ & $w_{7}$ & $w_{7}$ & $w_{7}$ & $w_{7}$ & $w_{7}$ & $w_{7}$ & $\theta$ & $w_{7}$ & $w_{7}$ \\
\hline$w_{8}$ & $w_{8}$ & $w_{8}$ & $w_{8}$ & $w_{8}$ & $w_{8}$ & $w_{8}$ & $w_{8}$ & $\theta$ & $w_{8}$ \\
\hline$w_{9}$ & $w_{9}$ & $w_{9}$ & $w_{9}$ & $w_{9}$ & $w_{9}$ & $w_{9}$ & $w_{9}$ & $w_{9}$ & $\theta$ \\
\hline
\end{tabular}

The multiplication table for the obtained Hilbert algebra is

\begin{tabular}{|l|l|l|l|l|l|l|l|l|l|}
\hline$\cdot$ & $\theta$ & $w_{2}$ & $w_{3}$ & $w_{4}$ & $w_{5}$ & $w_{6}$ & $w_{7}$ & $w_{8}$ & $w_{9}$ \\
\hline$\theta$ & $\theta$ & $w_{2}$ & $w_{3}$ & $w_{4}$ & $w_{5}$ & $w_{6}$ & $w_{7}$ & $w_{8}$ & $w_{9}$ \\
\hline$w_{2}$ & $\theta$ & $\theta$ & $w_{3}$ & $w_{4}$ & $w_{5}$ & $w_{6}$ & $w_{7}$ & $w_{8}$ & $w_{9}$ \\
\hline$w_{3}$ & $\theta$ & $w_{2}$ & $\theta$ & $w_{4}$ & $w_{5}$ & $w_{6}$ & $w_{7}$ & $w_{8}$ & $w_{9}$ \\
\hline$w_{4}$ & $\theta$ & $w_{2}$ & $w_{3}$ & $\theta$ & $w_{5}$ & $w_{6}$ & $w_{7}$ & $w_{8}$ & $w_{9}$ \\
\hline$w_{5}$ & $\theta$ & $w_{2}$ & $w_{3}$ & $w_{4}$ & $\theta$ & $w_{6}$ & $w_{7}$ & $w_{8}$ & $w_{9}$ \\
\hline$w_{6}$ & $\theta$ & $\mathbf{w}_{2}$ & $\mathbf{w}_{3}$ & $\mathbf{w}_{4}$ & $\mathbf{w}_{5}$ & $\theta$ & $w_{7}$ & $w_{8}$ & $w_{9}$ \\
\hline$w_{7}$ & $\theta$ & $\mathbf{w}_{2}$ & $\mathbf{w}_{3}$ & $\mathbf{w}_{4}$ & $\mathbf{w}_{5}$ & $w_{6}$ & $\theta$ & $w_{8}$ & $w_{9}$ \\
\hline$w_{8}$ & $\theta$ & $\theta$ & $\theta$ & $\mathbf{w}_{4}$ & $\mathbf{w}_{5}$ & $w_{6}$ & $w_{7}$ & $\theta$ & $w_{9}$ \\
\hline$w_{9}$ & $\theta$ & $\theta$ & $\mathbf{w}_{3}$ & $\theta$ & $\mathbf{w}_{5}$ & $w_{6}$ & $w_{7}$ & $w_{8}$ & $\theta$ \\
\hline
\end{tabular}

Using above notations, for $B=\left\{w_{6}, w_{7}, w_{8}, w_{9}\right\}$ and $A=\left\{w_{2}, w_{3}, w_{4}, w_{5}\right\}$, we remark that we obtain the initial code. We remark that $L_{1}=\left\{\theta, w_{2}, w_{3}, w_{4}, w_{5}, w_{6}, w_{7}, w_{9}\right\}, L_{2}=\left\{\theta, w_{2}, w_{3}, w_{4}, w_{5}, w_{6}, w_{7}, w_{8}\right\}$, $L_{3}=\left\{\theta, w_{2}, w_{3}, w_{4}, w_{5}, w_{6}, w_{8}, w_{9}\right\}, L_{4}=\left\{\theta, w_{2}, w_{3}, w_{4}, w_{5}, w_{7}, w_{8}, w_{9}\right\}$, $L_{5}=\left\{\theta, w_{2}, w_{3}, w_{4}, w_{6}, w_{7}, w_{8}, w_{9}\right\}, L_{6}=\left\{\theta, w_{2}, w_{3}, w_{5}, w_{6}, w_{7}, w_{8}, w_{9}\right\}$ are all maximal filters. Since $\bigcap_{i=1}^{6} L_{i}=\left\{\theta, w_{2}, w_{3}\right\}$, it results that this algebra is not semisimple and it is not local.

Example 4.2. We consider the binary block code $C=\{11111,01011,00111,00011,00001\}$. 
Since the codewords are lexicographically ordered, the obtained BCK algebra is $H=\{\theta, a, b, c, d\},(H, *)$, the obtained Hilbert algebra is $(H, \cdot)$ and have the multiplication given in the tables below.

\begin{tabular}{|l|l|l|l|l|l|}
\hline$*$ & $\theta$ & $a$ & $b$ & $c$ & $d$ \\
\hline$\theta$ & $\theta$ & $\theta$ & $\theta$ & $\theta$ & $\theta$ \\
\hline$a$ & $a$ & $\theta$ & $a$ & $\theta$ & $\theta$ \\
\hline$b$ & $b$ & $b$ & $\theta$ & $\theta$ & $\theta$ \\
\hline$c$ & $c$ & $c$ & $c$ & $\theta$ & $\theta$ \\
\hline$d$ & $d$ & $d$ & $d$ & $d$ & $\theta$ \\
\hline
\end{tabular}

\begin{tabular}{|l|l|l|l|l|l|}
\hline$\cdot$ & $\theta$ & $a$ & $b$ & $c$ & $d$ \\
\hline$\theta$ & $\theta$ & $a$ & $b$ & $c$ & $d$ \\
\hline$a$ & $\theta$ & $\theta$ & $b$ & $c$ & $d$ \\
\hline$b$ & $\theta$ & $a$ & $\theta$ & $c$ & $d$ \\
\hline$c$ & $\theta$ & $\theta$ & $\theta$ & $\theta$ & $d$ \\
\hline$d$ & $\theta$ & $\theta$ & $\theta$ & $\theta$ & $\theta$ \\
\hline
\end{tabular}

The proper filters are $L_{1}=\{\theta, a\}, L_{2}=\{\theta, b\}, L_{3}=\{\theta, a, b\}, L_{4}=\{\theta, a, b, c\}$. $L_{4}$ is the only maximal filter, therefore $(H, \cdot)$ is a local Hilbert algebra (see Theorem 3.12). $H$ is not a semisimple algebra.

Example 4.3. We consider the binary block code $C=\{1111,0100,0010,0001\}$. Since the codewords are lexicographically ordered, the obtained BCK algebra is $H=\{\theta, a, b, c\},(H, *)$, the obtained Hilbert algebra is $(H, \cdot)$ and have the multiplication given in the tables below.

\begin{tabular}{|l|l|l|l|l|}
\hline$*$ & $\theta$ & $a$ & $b$ & $c$ \\
\hline$\theta$ & $\theta$ & $\theta$ & $\theta$ & $\theta$ \\
$a$ & $a$ & $\theta$ & $a$ & $a$ \\
\hline$b$ & $b$ & $b$ & $\theta$ & $b$ \\
\hline$c$ & $c$ & $c$ & $c$ & $\theta$ \\
\hline
\end{tabular}

\begin{tabular}{|c|c|c|c|c|}
\hline$\cdot$ & $\theta$ & $a$ & $b$ & $c$ \\
\hline$\theta$ & $\theta$ & $a$ & $b$ & $c$ \\
\hline$a$ & $\theta$ & $\theta$ & $b$ & $c$ \\
\hline$b$ & $\theta$ & $a$ & $\theta$ & $c$ \\
\hline$c$ & $\theta$ & $a$ & $b$ & $\theta$ \\
\hline
\end{tabular}

The proper maximal filters are $L_{1}=\{\theta, a, b\}, L_{2}=\{\theta, b, c\}, L_{3}=\{\theta, a, c\} . L_{1}$, $L_{2}, L_{3}$ are maximal filters and $L_{1} \cap L_{2} \cap L_{3}=\{e\}$, therefore $(H, \cdot)$ is not a local Hilbert algebra but it is a semisimple algebra (see Theorem 3.11).

Conclusions. In the papers (Jun, Song, 2011), (Flaut, 2015), (Borumand Saeid, Fatemidokht, Flaut, Kuchaki Rafsanjani, 2015), some connections between BCK-algebras and binary block codes were described. In this paper we make some connections between Hilbert algebras and binary block codes via BCK-algebra defined by relation (2.1). In this way, using codes, we found examples of classes of semisimple Hilbert algebras and classes of local Hilbert algebras. As further research, we will try to find answers to the reverse problem, namely, how properties of BCK-algebras, BCI-algebras, Hilbert algebras can influence the properties of binary block codes.

\section{References}

Borumand Saeid, A., Fatemidokht, H., Flaut, C., Kuchaki Rafsanjani, M., (2015), On Codes based on BCK-algebras, arxiv, 13 pages. 
D. Buşneag, On the maximal deductive system of a bounded Hilbert algebra, (1987), Bull. Math. Soc. Sci. Math. Roumanie, 31(1)(79), pp. 9-21.

Chajda, I., Halaš, R., Jun, Y.B., (2002), Annihilators and deductive systems in commutative Hilbert algebras, Comment. Math. Univ. Carolin., 43(3), pp. 407-417.

Dan, C., (2008), Hilbert algebras of Fractions, Int. J. Math. and Math. Sci., 2009, Article ID 589830 (16 pages).

Diego A., (1966), Sur les algébras de Hilbert, Ed. Hermann, Colléction de Logique Math. Serie A, 21, pp. 1-52.

Dudek, W.A., (1999), On embedding Hilbert algebras in BCK-algebras, Mathematica Moravica, 3, pp. 25-28.

Flaut, C., (2015), BCK-algebras arising from block codes, J. Intell. Fuzzy Syst., 28(4), pp. 1829-1833.

Jun, Y. B., Song, S. Z., (2011), Codes based on BCK-algebras, Inform. Sciences., 181, pp. 5102-5109.

Piciu, D., Buşneag, C., (2010), The localization of commutative (unbounded) Hilbert algebras, Math. Rep., 3(12)(62), 16 pages. 